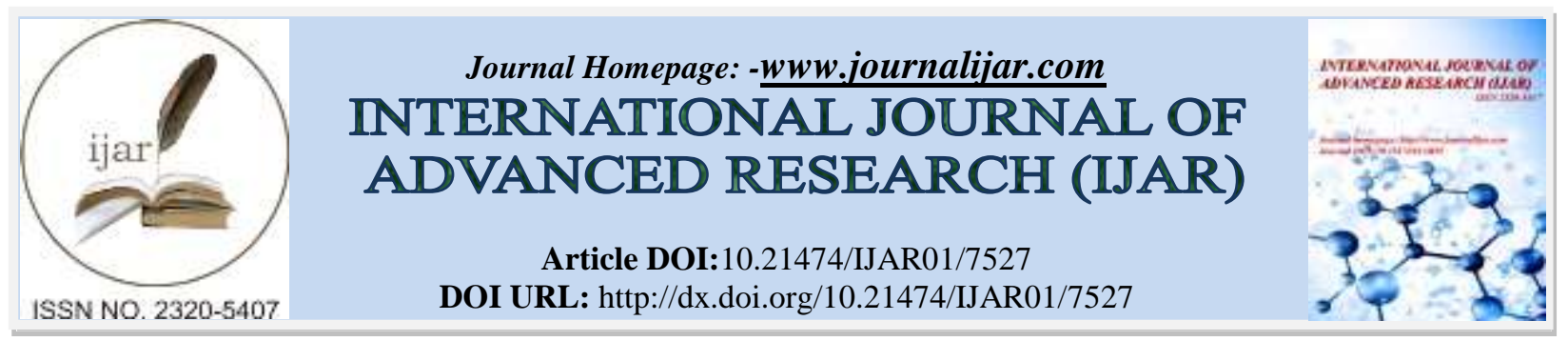

RESEARCH ARTICLE

\title{
NEUROBRUCELLOSIS: A REPORT OF TWO CASES.
}

Mohamed Amine MNAILI, Nabil Abida, Youssouf Benmouh, Amine Raggabi and Ahmed Bourazza. Service de Neurologie, Hôpital Militaire d'instruction Mohammed V Rabat.

\section{Manuscript Info}

Manuscript History

Received: 10 June 2018

Final Accepted: 12 July 2018

Published: August 2018

Keywords:-

Brucellosis, Neurobrucellosis,

Meningitis.

\begin{abstract}
Brucellosis is a ubiquitous zoonosis particularly affecting the Mediterranean countries and the Middle East. Its clinical presentation is quite heterogeneous and diagnosis requires a high index of suspicion in patients from endemic areas. The radiological abnormalities of neurobrucellosis are also variable and may mimic other inflammatory, infectious or vascular diseases. We present two cases of neurobrucellosis with varying clinical involvement from south of Morocco. The results of lumbar puncture, magnetic resonance imaging and especially serology have led to the conclusion of neurobrucellosis. Neurobrucellosis is a serious disease whose prognosis depends on the early diagnosis and treatment.
\end{abstract}

Copy Right, IJAR, 2018,. All rights reserved.

\section{Introduction:-}

La brucellose ou la fièvre de Malte est une anthropozoonose décrite d'abord chez les animaux domestiques puis chez l'homme. Sa pathologie dépend largement du réservoir animal. Les espèces les plus incriminées en clinique sont Brucella abortus, Brucella melitensis et Brucella suis. La brucellose humaine est l'anthropozoonose, la plus répandue dans le monde. Elle demeure endémique dans certains pays du bassin méditerranéen, au Moyen Orient, en Asie de l'Ouest et dans certaines régions d'Afrique et d'Amérique Latine. Les manifestations neurologiques de la brucellose sont polymorphes et peuvent se manifester d'une manière aigue, subaigu ou chronique. En cas d'infection aigue, elles sont non spécifiques, entrainant des céphalées, une asthénie et des myalgies. Dans les atteintes chroniques, les manifestations neurologiques sont variées, ce qui peut rendre le diagnostic difficile. Nous rapportons deux cas de neurobrucellose provenant du sud du pays qui ont atterrie dans notre structure avec des présentations cliniques différentes. Nous soulignons également que ce diagnostic doit être évoqué devant toute manifestation neurologique inexpliquée.

\section{Observation 1:}

Un jeune homme de 17 ans, vivant dans le sud du pays, sans antécédents pathologiques notables s'est présenté aux urgences dans un tableau de céphalées chroniques évoluant depuis 9 mois associé à quelques épisodes de vomissements. L'interrogatoire avait trouvé une notion de fièvre intermittente avec un amaigrissement non chiffré mais sans trouble visuel ni lourdeur des membres ni de trouble de l'équilibre. Il avait reçu une antibiothérapie empirique durant les épisodes de fièvre sans réelle amélioration. L'examen clinique trouvait un patient conscient, fébrile à 39 avec une nuque raide, sans déficit sensitivo moteur mais des reflex ostéotendineux vifs aux membres inférieurs.

L'IRM cérébral avait objectivé une prise de contraste léptoméningé diffuse (figure 1). L'étude du liquide céphalorachidien (LCR) avait révélé un liquide jaune citrin, avec 100 globules blancs/mm3 (73\% de lymphocytes), 
une protéinorachie à $4,11 \mathrm{~g} / \mathrm{L}$ et une glycorachie à $0,11 \mathrm{~g} / \mathrm{L}$ (glycémie concomitante à $1,08 \mathrm{~g} / \mathrm{L}$ ). La coloration de Gram était normale. L'analyse des antigènes solubles avait éliminé d'autres pathogènes de la méningite. La recherche GeneXpert dans le LCR était négative, excluant le complexe mycobacterium tuberculosis.

Le test de Rose Bengale (Brucellose slide BioMerieux® France) était positif dans le sérum et le LCR. Le test de Wright dans le LCR (brucella wright biorad®, France) était positif avec un titre de 640. Les sérologies du VIH, de la syphilis, des hépatites $\mathrm{B}$ et $\mathrm{C}$, de la maladie de Lyme étaient négatifs. Les examens biologiques comprenant une numération formule sanguine, un ionogramme sanguin ainsi qu'un bilan thyroïdien et immunologique étaient sans anomalie notable.

Un bilan cardiovasculaire comprenant une ETT et ETO à la recherche d'endocardite avec végétations était normal. L'électroencéphalographie avait montré des pointes lentes en temporal gauche. L'examen ophtalmologique était normal. Le scanner thoraco abdomino pelvien avait objectivé des ganglions lymphatiques dans les chaînes coronaires stomachiques et les régions coeco colo appendiculaire ainsi d'un discret tassement antérieur du corps vertébral de D11 sans signes d'instabilité.

Le diagnostic de la neurobrucellose fut retenu et un traitement par antibiotiques antibrucelliens (Doxycycline 200mg/jr et Rifampicine $900 \mathrm{mg} / \mathrm{jr}$ ) a été démarré et maintenu pendant 3 mois avec une évolution clinique et biologique favorable.

\section{Observation 2:-}

Patiente de 49 ans sans antécédents pathologiques notables ayant présenté depuis 6 mois de façon progressive une lourdeur des 2 membres inférieurs sans manifestations sensitives subjectives ni troubles sphinctériens ni atteinte des membres supérieurs. Cette symptomatologie s'accompagnait d'un vertige rotatoire avec hypoacousie bilatérale sans troubles visuels. Le tout évoluait dans un contexte d'apyrexie et de conservation de l'état général.

L'examen clinique avait trouvé une patient consciente, bien orienté, apyrétique, sans syndrome méningé avec une paraparésie spastique sans troubles sphinctériens ni troubles de l'équilibre. L'audiométrie avait objectivé une surdité mixte à prédominance perceptionnelle.Il n'existait pas, en revanche, de signes d'atteinte vestibulaire, avec une vidéonystagmographie n'objectivant pas de signes d'atteinte centrale.

L'IRM cérébrale avait objectivé des lésions en plage de la substance blanche bilatérales autour des ventricules latéraux, hyperintenses en séquences pondérés T2 et Flair, avec une prise de contraste leptoméningée. (Figure 2)

L'étude du liquide céphalorachidien (LCR) avait montrait un liquide clair, avec 130 globules blancs/mm3 (80 \% de lymphocytes), une protéinorachie à $4,95 \mathrm{~g} / \mathrm{L}$ et une glycorachie à $0,30 \mathrm{~g} / \mathrm{L}$ (glycémie concomitante à $1,01 \mathrm{~g} / \mathrm{L}$ ). L'examen direct et les cultures pour bactéries, tuberculose et mycoses étaient stériles. La recherche GeneXpert dans le LCR était négative.

Le test de Rose Bengale était positif dans le sérum et le LCR. Le test de Wright dans le LCR était positif avec un titre de 640. Une échocardiographie transthoracique et transœsophagienne ainsi qu'un écho-doppler des vaisseaux du cou étaient normaux.

Le diagnostic de la neurobrucellose fut retenu et un traitement par antibiotiques antibrucelliens (Doxycycline 200mg/jr et Rifampicine $900 \mathrm{mg} / \mathrm{jr}$ )) a été démarré avec une évolution clinique partielle avec amélioration de la marche et de la force musculaire au niveau des membres inférieurs mais persistance de l'hypoacousie et normalisation des données du LCR

\section{Discussion:-}

L'atteinte du système nerveux est rare dans la brucellose. Elle peut survenir à la phase aigue ou plusieurs mois plus tard, avec une grande diversité des modes de présentation clinique. Cela explique que le diagnostic de neurobrucellose chronique est difficile et souvent retardé en dehors des régions endémiques. [1] La brucellose animale, quoique maintenant rare, est endémique au Maroc. Certaines habitudes alimentaires (plats à base de viande crue, consommation de lait frais non bouilli, fromages à base de lait cru) contribuent à la contamination humaine.En tenant compte de nos données épidémiologiques, tout en sachant que les gens du sud du Maroc boivent le lait de 
chameau cru comme coutume, ainsi que dans l'histoire des 2 patients, nous avions noté que le père du premier patient et la sœur de la deuxième patiente ont été traité pour une brucellose, le diagnostic a été rapidement évoqué.

La physiopathologie des différentes phases de la neurobrucellose n'est pas complètement élucidée. Dans les formes aigues, le germe a un effet direct sur le système nerveux mais il peut rester longtemps à l'intérieur des corps cellulaires et, à l'occasion d'une baisse de l'immunité, il peut proliférer et entrainer des manifestations subaigües ou chroniques. Dans les formes chroniques, ce mécanisme s'associerait à un dysfonctionnement immunitaire, comme le suggèrent les rares cas de démyélinisation du système nerveux central ou périphérique comme le cas de la deuxième patiente [2]

La fréquence des manifestations neurologiques de la brucellose est de $10 \%$ des cas. Il peut s'agir de méningite, de méningo-encéphalite aigue ou chronique, d'hypertension intracrânienne, de méningomyélite, de compression médullaire par spondylodiscite, d'atteinte de nerfs crâniens ou de polyradiculonévrite. Des accidents vasculaires cérébraux artériels ou veineux ont aussi été rapportés. [3]

La méningoencéphalite peut se révéler précocement par un tableau clinique aigu ou avoir une évolution prolongée [4]. Dans sa forme chronique, les symptômes peuvent précéder le diagnostic de plusieurs mois. C'était le cas de nos deux patients chez qui le diagnostic de méningite lymphocytaire a été fait plus de 6 mois après le début de la symptomatologie.

Le diagnostic radiologique de la neurobrucellose est basé sur l'existence d'une lésion du SNC non expliquée par une autre pathologie neurologique [14]. Seulement $45 \%$ des patients atteints de neurobrucellose présentent des anomalies radiologiques. Les résultats radiologiques peuvent être regroupés en 4 catégories: (i) Lésions inflammatoires: abcès cérébral, granulomes et arachnoïdite, lésions leptoméningées, atteintes basales des méningés ou des nerfs crâniens, (ii) Atteinte de la substance blanche avec ou sans lésions démyélinisantes, (ii) lésions vasculaires d'une maladie cérébrale ischémique chronique, (iv) hydrocéphalie, œdème cérébral [14].Chez nos patients, l'un avait une prise de contraste léptoméningée diffuse ainsi que l'autre des lésions démyélinisantes sus tentorielles. [5].

Dans la neurobrucellose, le LCR est normal chez 84,6\% [6]. Nos deux patients avaient une méningite lymphocytaire avec une hyperprotéinorachie et une hypoglycorachie. Ces résultats peuvent bien corréler avec le diagnostic de tuberculose, en particulier dans notre pays où la tuberculose est plus endémique que la brucellose. Mais la présentation chronique était contre cette hypothèse, une tuberculose neuromémingé pourrait être fatale si le traitement est retardé. De plus, le test de geneXpert était négatif. Ainsi, les tests d'agglutination sérique de Rose Bengal et Wright étaient positifs dans le LCR. Ceci concorde avec les données la littérature puisque les tests sérologiques dans le LCR peuvent être positifs dans $77 \%$ des cas. [6]

Les diagnostics différentiels de la neurobrucellose sont nombreux car le pathogène ne donne pas de syndrome clinique typique ou de modification spécifique du LCR. Un grand nombre d'investigations habituellement utilisées dans le diagnostic de la brucellose donnent souvent des résultats négatifs [6]. En effet, les résultats faussement négatifs des tests de séro-agglutination (SAT) peuvent être obtenus en présence d'anticorps bloquants ou d'une absence d'agglutination dans de faibles dilutions sériques, phénomène appelé prozone; Le test de Coombs augmente la sensibilité de la SAT [7].

La spécificité de l'hémoculture dans le diagnostic de la brucellose est comprise entre 17\% et $85 \%$ [8]. Elle était négative chez nos deux patients. En conséquence, la maladie doit être évoquée chez tous les patients qui développent des symptômes neurologiques inexpliqués, en particulier ceux qui vivent dans des zones endémiques. Dans nos cas, d'autres étiologies ont été évoquées, à savoir la borréliose et la neurosyphilis qui avaient été exclues par des tests appropriés.

Le traitement de la neurobrucellose est loin d'être codifié. Il repose sur des antibiotiques actifs ayant une bonne diffusion intracellulaire et à travers la barrière hémato encéphalique. Ce traitement doit être précoce et inclure deux à trois antibiotiques synergiques et spécifiques. Ils sont représentés par des cyclines (doxycycline, minocycline), à une dose de $200 \mathrm{mg}$ par jour (adultes) associés à la rifampicine à raison de $20 \mathrm{mg} / \mathrm{kg} /$ jour chez les enfants et 600 à $1200 \mathrm{mg} /$ jour chez les adultes ou un aminoglycoside (streptomycine en particulier) pendant les 6 premières semaines de traitement [9]. 
Autres antibiotiques tels que les Amphenicols, la Pénicilline A et le Cotrimoxazole ont une activité discutable in vivo. La durée du traitement pour le neurobrucellose dépend du stade de l'infection. Dans les formes secondaires notamment la méningo-encéphalite, la durée ne doit pas être inférieure à 3 mois. Le taux de rechute est inférieur à $5 \%$ [10]. L'Ajout de lévofloxacine au traitement cycline-rifampicine peut augmenter son efficacité en terme de diminution du taux de rechute de la maladie

\section{Conclusion:-}

La brucellose est une infection multi-systémique, avec différentes présentations cliniques. La neurobrucellose est parmi les formes les plus graves de l'infection. Ses symptômes cliniques et ses signes neuroradiologiques sont généralement atypiques et peuvent être confondu avec d'autres pathologies neurologiques; ce qui explique le retard diagnostic et thérapeutique. Dans les zones endémiques, la neurobrucellose devrait être évoqué devant toute symptomatologie neurologique inexpliquée.

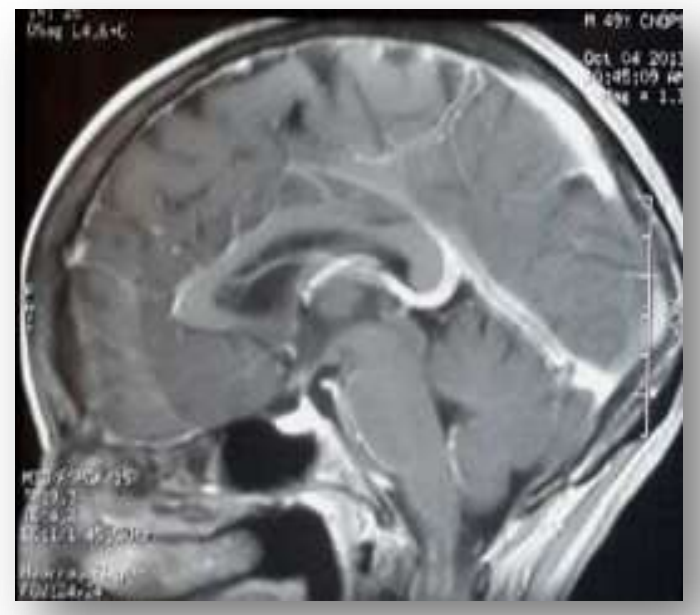

Figure 1: (patient 1) : IRM cérébrale en séquence T1 injectée en coupe sagittale montrant une prise de contraste méningée intense
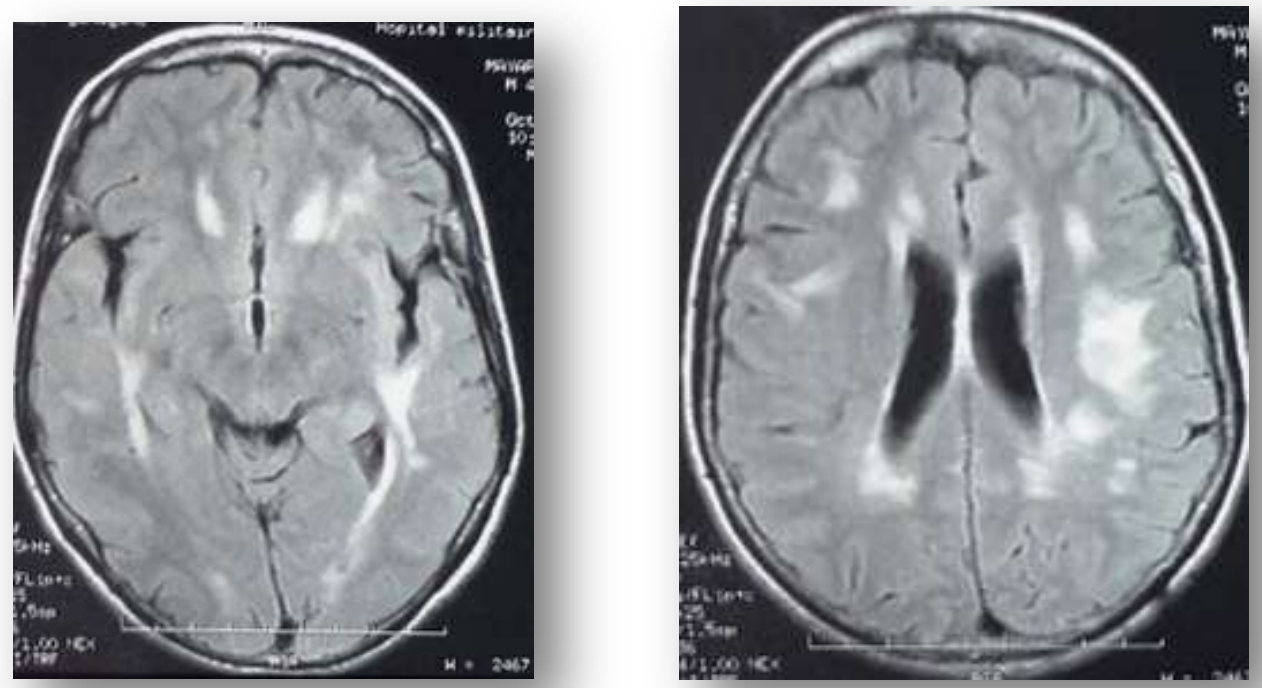

Figure 2 (patiente 2): IRM cérébrale coupes axiales séquence Flair montrant des hyper signaux péri-ventriculaires 


\section{References:-}

1. Sola M, Giron C, Blanco A, Martin U, Moreno M, Cardiel B. Neurobrucellosis. A report of 13 cases. Neurologia 1995;10:375-9.

2. Morin B, Tournilhac M, Grellet C, Rey M. La neurobrucellose. Nouv Presse Med 1980;9:871-5.

3. Awada A, Korri H, Issa Z, Ali Y, Beaini M. Paraparésie et surdité progressives avec leuco-encéphalopathie révélant une neurobrucellose chronique. Revue neurologique. 2011Feb; 167(2):181-4.

4. Mc Lean DR, Russell N, Khan Y. Neurobrucellosis: clinical and therapeutic features. Clin Infect Dis 1992;15:582-90.

5. Erdem H, Senbayrak S, Meriç K, Batirel A, Karahocagil MK, Hasbun R, et al. Cranial imaging findings in neurobrucellosis: results of Istanbul-3 study. Infection 2016;44(October (5)):623-31. http://dx.doi.org/10.1007/s15010-016-0901-3. Epub 2016 May 2

6. Haji-Abdolbagi M, Rasooli-Nejad M, Jafari S, Hasibi M, Soudbakhsh A. Clinical and laboratory findings in neurobrucellosis: review of 31 cases. Arch Iran Med. 2008;11(January (1)):21-5.

7. Guven GS, Cakir B, Oz G, Tanriover MD, Turkmen E, Sozen T. Could remembering the prozone phenomenon shorten our diagnostic journey in brucellosis? A case of Brucella spondylodiscitis. Rheumatol Int 2006;26:9335.

8. Chelli Bouaziz M, Ladeb MF, Chakroun M, Chaabane S. Spinal brucellosis: a review. Skeletal Radiol 2008;37:785-90.

9. World Health Organization. Brucellosis in humans and animals. Treatment of brucellosis in humans 7. WHO/CDS/EPR; 2006. p. 36-8.

10. Ben Hamouda I, Gouider R, Mrabet A. EMC Neurobrucellose 2007:50. http://dx.doi.org/10.1016/S02460378(07)43860-X. 